\title{
PARAMETER ESTIMATION ALGORITHMS FOR IONOSPHERIC CHANNELS
}

\author{
J.L. Sanz-González*, S. Zazo-Bello*, I. A. Pérez-Álvarez ${ }^{\dagger}$, J. López-Pérez ${ }^{\dagger}$ \\ * Universidad Politécnica de Madrid (UPM), Dpto. SSR, ETSI de Telecomunicación-UPM, Ciudad Universitaria, 28040 - \\ Madrid, Spain, Tel.: +34-913367226, e-mail: jlsanz@gcs.ssr.upm.es \\ $\dagger$ Universidad de Las Palmas de Gran Canaria, CeTIC, Dpto. Señales y Comunicaciones, Campus de Tafira, 35017-Las \\ Palmas, Spain, Tel.: +34-982457362, e-mail: iperez@dsc.ulpgc.es
}

Keywords: Ionospheric channel, path delay, Doppler spread.

\begin{abstract}
The principal parameters of a multipath channel are the delay and the gain coefficient of each path, and the number of significant paths. We develop a methodology for estimating path-delays and path-coefficients and applied it to the ionospheric channel estimation. Also, if path coefficients are stationary random processes, the power spectral density of the coefficients can be estimated from measured data of real channels. Finally, some parameter estimations for real narrowband HF ionospheric channels are shown, where several rays are detected with a Doppler spread about $0.2 \mathrm{~Hz}$.
\end{abstract}

\section{Introduction}

As is well known, a radio HF link by ionospheric reflection is an important alternative to satellite link when cost, confidence and security of communications are considered. HF communication systems were used in military applications due to the technological challenge for strategic communications, and as an attractive alternative to other radio communications. Presently, there is a large expertise in this field for both civil and military applications.

Watterson et al. [1] analyzed HF ionospheric channels from measured data and estimated the parameters of a transversal filter (tapped delay line) with time-variant coefficients, concluding that for narrowband channels (up to $12 \mathrm{KHz}$ ) the hypotheses about the Gaussian characteristic of the coefficients (Gaussian processes and Gaussian spectra) is fulfilled. In [2] a new simple narrowband HF ionospheric channel model is introduced by modelling independently delay and Doppler effects, and in $[3,4]$ models for wideband (up to $1 \mathrm{MHz}$ ) HF ionospheric channels are presented.

In a general multipath channel model, the path delays and path coefficients are time-dependent (model useful for both narrow- and wide- band channels). The problem is the reliable estimation of the path-delays and path-coefficients from measured data. In fact, we have a matrix of data (twodimensional data) that corresponds to frequency and time, as it can be seen later. Then, the problem is reduced to the estimation of sinusoidal signals (amplitudes, phases and frequencies). This is a classical problem in spectral analysis
[5, Chap. 16], but the well-known Prony's method doesn't work adequately under noisy data [5, pp. 1193], and other methods based on eigen-structures are not applied here due to the non-stationary character of the problem.

In this paper, we propose a new methodology for this estimation problem based on, first to estimate path delays and, then, to estimate path coefficients after incorporating delay estimations. Algorithms for solving this problem have been developed by the authors of this paper and some results will be shown later. In Section 2, we define the model to be used; in Section 3, we describe the algorithm in general terms; in Section 4, we show estimation results for real ionospheric channels. Finally, Section 5 summarizes the main conclusion of the paper.

\section{Ionospheric channel as a multipath channel}

A linear time-variant system can be characterized by the convolution integral as follows [6]

$$
y(t)=\int_{-\infty}^{\infty} h(\tau ; t) x(t-\tau) d \tau
$$

where $x(t)$ is the system input, $y(t)$ is the system output and $h(\tau ; t)$ is the unit impulse response of the time-variant system, i.e. the response of the system at time " $t$ " to a unit impulse (a Dirac delta function) located at time " $t-\tau$ ". If $h(\tau ; t)$ does not depend on " $t$ ", we have the well-known linear time-invariant system. According to [7], the impulse response $h(\tau ; t)$ of a general multipath channel may be modelled by

$$
h(\tau ; t)=\sum_{k=1}^{K} a_{k}(t) g\left(\tau-\tau_{k}(t)\right)
$$

where $K$ is the number of paths between the transmitter and the receiver, $a_{k}(t)$ is the complex coefficient of the $k$ th-path, $\tau_{k}(t)$ is the corresponding path delay, $g(t)$ is the equivalent impulse response of the transmitter filters. It is assumed the corresponding equivalent low-pass complex-envelope signals. Now, if the input signal $x(t)=e^{j \omega_{0} t}$, from (1) we have $y(t)=H\left(j \omega_{0} ; t\right) \cdot e^{j \omega_{0} t}$, where $H(j \omega ; t)$ is the transfer function of the multipath channel, i.e. 


$$
H(j \omega ; t)=\sum_{k=1}^{K} a_{k}(t) e^{-j \omega \cdot \tau_{k}(t)} \cdot G(j \omega)
$$

where $G(j \omega)$ is the equivalent transfer function of the transmitter filters.

Our purpose is to estimate complex coefficients $a_{k}(t)$ and delays $\tau_{k}(t)$ from noisy measures of $H(j \omega ; t)$. Consequently, $H(j \omega ; t)$ will be sampled in its two variables, i.e. $\omega_{m}=\omega_{1}+m \cdot \Delta \omega, m=1,2, \ldots, M$, and $t_{n}=t_{1}+n \cdot \Delta t, n=1$, $2, \ldots, N$, in such a way that $H\left(j \omega_{m} ; t_{n}\right), m=1,2, \ldots, M$, and $n=1,2, \ldots, N$, represent $H(j \omega ; t)$. Also, it is supposed that $G\left(j \omega_{m}\right) \approx 1$ for $m=1,2, \ldots, M$, i.e. the frequency samples are inside the transmitter passband. (If $G(j \omega) \neq 1$, we divide both sides of (3) by $G(j \omega))$.

\section{Algorithms to estimate multipath parameters}

For the parameter estimation, a good criterion is the minimization of the quadratic error $\varepsilon_{n}\left(\mathbf{a}_{n}, \boldsymbol{\tau}_{n}\right)$, defined by

$$
\varepsilon_{n}\left(\mathbf{a}_{n}, \boldsymbol{\tau}_{n}\right)=\sum_{m=1}^{M}\left|H\left(j \omega_{m} ; t_{n}\right)-\sum_{k=1}^{K} a_{k}\left(t_{n}\right) e^{-j \omega_{m} \cdot \tau_{k}\left(t_{n}\right)}\right|^{2}
$$

where $|\cdot|$ means "magnitude of • ", and column vectors $\mathbf{a}_{\mathbf{n}}=\left[a_{1}\left(t_{n}\right), a_{2}\left(t_{n}\right), \ldots, a_{K}\left(t_{n}\right)\right]^{T}, \boldsymbol{\tau}_{\mathbf{n}}=\left[\tau_{1}\left(t_{n}\right), \tau_{2}\left(t_{n}\right), \ldots, \tau_{K}\left(t_{n}\right)\right]^{T}$

A standard (gradient, genetic, etc) minimization algorithm applied to (4) does not work adequately because of the large amount of local minima. Also, the global minimum of (4) is very critical and sensitive with respect to vector $\tau_{n}$. Note that if vector $\boldsymbol{\tau}_{n}$ is known, vector $\mathbf{a}_{n}$ that minimize (4) can be solved from the well-known linear equation

$$
\mathbf{a}_{n}=\left(\mathbf{M}_{e}^{*} \cdot \mathbf{M}_{e}\right)^{-1} \cdot \mathbf{M}_{e}^{*} \cdot \mathbf{h}_{n}
$$

where matrix $\mathbf{M}_{e}^{*}$ is the complex-conjugate transpose of

$$
\mathbf{M}_{e}=\left[\begin{array}{cccc}
e^{-j \omega_{1} \tau_{1}\left(t_{n}\right)} & e^{-j \omega_{1} \tau_{2}\left(t_{n}\right)} & \cdots & e^{-j \omega_{1} \tau_{K}\left(t_{n}\right)} \\
e^{-j \omega_{2} \tau_{1}\left(t_{n}\right)} & e^{-j \omega_{2} \tau_{2}\left(t_{n}\right)} & \cdots & e^{-j \omega_{2} \tau_{K}\left(t_{n}\right)} \\
\vdots & \vdots & \cdots & \vdots \\
e^{-j \omega_{M} \tau_{1}\left(t_{n}\right)} & e^{-j \omega_{M} \tau_{2}\left(t_{n}\right)} & \cdots & e^{-j \omega_{M} \tau_{K}\left(t_{n}\right)}
\end{array}\right]
$$

for each $t_{n}, n=1,2, \ldots, N$, and column vector $\mathbf{h}_{n}$ is also giving as $\mathbf{h}_{n}=\left[h_{1 n}, h_{2 n}, \ldots, h_{M n}\right]^{T}$, being $h_{m n}=H\left(j \omega_{m} ; t_{n}\right)$; $m=1,2, \ldots, M$. Therefore, the problem is to estimate the delay vector $\boldsymbol{\tau}_{n}$.

\subsection{First Estimation of path delays.}

Suppose we know $H\left(j \omega ; t_{n}\right)$ in the passband of the measurement system, and that $W_{B}(j \omega)$ is a frequency window narrower than the passband system; now, taking the inverse Fourier transform of $H\left(j \omega ; t_{n}\right) \cdot W_{B}(j \omega)$ and considering the modulus of the result (in the $\tau$-domain), we have

$$
\left|h\left(\tau ; t_{n}\right) * w_{B}(\tau)\right|=\left|\sum_{k=1}^{K} a_{k}\left(t_{n}\right) w_{B}\left(\tau-\tau_{k}\left(t_{n}\right)\right)\right|
$$

where $w_{B}(\tau)$ is the inverse Fourier transform of $W_{B}(j \omega)$, and "*" means convolution operation. Therefore, to detect adequately $\tau_{k}$ by a peak detector, the separation among delays $\tau_{k}, k=1,2, \ldots, K$, must be greater than the time window length $w_{B}(\tau)$ which is the inverse value of the frequency window length $W_{B}(j \omega)$; also, the side-lobe aliasing and the noise of the measured data perturb the estimation of $\tau_{k}, k=1,2, \ldots, K$. It is important to use an adequate frequency window $W_{B}(j \omega)$ for minimizing timealiasing and noise effects on (7). In our application we have used a Kaiser window for $W_{B}(j \omega)$ with a shape parameter $\beta=2$ (see, for example, [5]). For separations among pathdelays grater than the length of the time window $w_{B}(\tau)$, the peak detector over (7) is a quasi-optimum detector under white Gaussian noise, because the referred approach is related to the matched filter applied to each $k$ th-ray.

In order to estimate close $\tau_{k}(k=1,2, \ldots, K)$ in (7) at time $t_{n}$, we use a threshold for detecting possible intervals of $\tau$ values that contain several close $\tau_{k}$ 's. The threshold should be estimated from the noise level, and set adequately in order to prevent noise-peak detections. If the length of a detected interval is lower than the window length of $w_{B}(\tau)$, it is supposed that there is one $\tau_{k}$ in the middle of the referred interval. If the length of the detected interval is greater than a $w_{B}(\tau)$ window length, and lower than two time-window lengths, then it is supposed that there are two $\tau_{k}$ 's out of $K$ in the referred interval, and so on. Therefore, multiple $\tau_{k}$ 's can be estimated inside a detected peak-interval, depending on the interval length, resulting in a delay resolution lower than a half of the time-window length of $w_{B}(\tau)$. Furthermore, for very close paths it is difficult to detect the paths with low intensity (say, lower than a tenth of the largest intensity). For these cases, we have proposed to remove all the strongest rays after they have been estimated (i.e. subtracting them from the data), and then to estimate successively the lower rays.

\subsection{First Estimation of path coefficients.}

Once the delays $\tau_{k}(k=1,2, \ldots, K)$ have been estimated, where $K$ is the maximum number of detected $\tau_{k}$ 's, the corresponding coefficients $a_{k}$ can be computed from (5). Note that in fact, we have $\tau_{k}\left(t_{n}\right)$ and $a_{k}\left(t_{n}\right)$ for $k=1,2, \ldots, K$, at time instant " $t_{n}$ ", and we have to repeat the estimations for all time instants $t_{n}, n=1,2, \ldots, N$. Finally, a precise estimation 
of vectors $\mathbf{a}$ and $\boldsymbol{\tau}$ for a fixed $t_{n} \quad(n=1,2, \ldots, N)$ can be done if we apply an optimization gradient algorithm after the first estimation given above. The details are given below.

\subsection{Refining estimations of delays and coefficients.}

Suppose we have an initial approximation $\boldsymbol{\tau}^{(0)}$ to $\boldsymbol{\tau}_{n}$ that corresponds to the global minimum of equation (4), from equation (5) we compute an initial approximation $\mathbf{a}^{(0)}$ of $\mathbf{a}_{n}$ that corresponds to the global minimum of equation (4), i.e.

$$
\mathbf{a}^{(0)}=\left(\mathbf{M}_{e}^{*(0)} \mathbf{M}_{e}^{(0)}\right)^{-1} \mathbf{M}_{e}^{*(0)} \mathbf{h}
$$

where matrix $\mathbf{M}_{e}^{(0)}$ is computed from (6) for $\boldsymbol{\tau}_{n}=\boldsymbol{\tau}^{(0)}$ For $i=1,2, \ldots, I$ compute the following formulas:

$$
\begin{aligned}
\boldsymbol{\tau}^{(i)} & =\boldsymbol{\tau}^{(i-1)}-\mu \cdot \nabla_{\boldsymbol{\tau}}^{(i-1)}(\varepsilon(\mathbf{a}, \boldsymbol{\tau})) \\
\mathbf{a}^{(i)} & =\left(\mathbf{M}_{e}^{*(i)} \mathbf{M}_{e}^{(i)}\right)^{-1} \mathbf{M}_{e}^{*(i)} \mathbf{h}
\end{aligned}
$$

where $\boldsymbol{\tau}^{(i)}$ is the value of $\boldsymbol{\tau}$ for the iteration $i, \mu$ is the step parameter that controls the convergence speed and the stability of the algorithm, and column vector $\nabla_{\tau}^{(i)}(\varepsilon(\mathbf{a}, \boldsymbol{\tau}))$ is the gradient of $\varepsilon(\mathbf{a}, \boldsymbol{\tau})$ with respect to vector $\boldsymbol{\tau}$ for $\mathbf{a}=\mathbf{a}^{(i)}$ and $\boldsymbol{\tau}=\boldsymbol{\tau}^{(i)}$, i. e. $\nabla_{\boldsymbol{\tau}}^{(i)}(\varepsilon(\mathbf{a}, \boldsymbol{\tau}))=\left[\nabla_{\boldsymbol{\tau}}(\varepsilon(\mathbf{a}, \boldsymbol{\tau}))\right]_{\mathbf{a}=\mathbf{a}^{(i)}, \boldsymbol{\tau}=\boldsymbol{\tau}^{(i)}}$, being

$$
\begin{aligned}
& \nabla_{\boldsymbol{\tau}}(\varepsilon(\mathbf{a}, \boldsymbol{\tau}))=2 \mathrm{R}_{\mathrm{e}}\left\{\sum_{m=1}^{M}\left(\frac{\partial e_{m}(\mathbf{a}, \boldsymbol{\tau})}{\partial \boldsymbol{\tau}}\right)^{*} \cdot e_{m}(\mathbf{a}, \boldsymbol{\tau})\right\} \\
& =2 \mathrm{I}_{\mathrm{m}}\left\{\left(\mathbf{M}_{e} \operatorname{diag}(\mathbf{a})\right)^{*} \operatorname{diag}(\boldsymbol{\omega})\left(\mathbf{h}_{n}-\mathbf{M}_{e} \cdot \mathbf{a}\right)\right\}
\end{aligned}
$$

where $\mathrm{R}_{\mathrm{e}}\{\cdot\}$ and $\mathrm{I}_{\mathrm{m}}\{\cdot\}$ mean real and imaginary parts, and

$$
e_{m}(\mathbf{a}, \boldsymbol{\tau})=H\left(j \omega_{m} ; t_{n}\right)-\sum_{k=1}^{K} a_{k} e^{-j \omega_{m} \cdot \tau_{k}}
$$

and matrix $\mathbf{M}_{e}{ }^{(i)}$ is computed from (6) for $\boldsymbol{\tau}=\boldsymbol{\tau}^{(i)}$ and $\boldsymbol{\omega}=\left(\omega_{1}, \omega_{2}, \ldots, \omega_{M}\right)$. The stopping value $I$ (last iteration) is considered when $\boldsymbol{\tau}^{(I)}$ is close enough to $\boldsymbol{\tau}^{(I-1)}$.

It is important to note that the estimation refinement is possible if the measured data are not too noisy (e.g. from our simulations: signal-to-noise ratio greater than $10 \mathrm{~dB})$; otherwise, the first estimation of path delays given above in Subsection 3.1 can not be improved by the refining equation (9). Our algorithm realizes a test to accept or reject the computed refinements according to the computed errors for estimated values $\mathbf{a}=\mathbf{a}^{(i)}$ and $\boldsymbol{\tau}=\boldsymbol{\tau}^{(i)}$. After the path delays $\tau_{k}\left(t_{n}\right)$ and the path coefficients $a_{k}\left(t_{n}\right)$ for $k=1,2, \ldots, K$, have been estimated at time instant " $t_{n}$ ", we have to repeat the estimations for all time instants $t_{n}, n=1,2, \ldots, N$. Also, we can plot $\tau_{k}\left(t_{n}\right)$ versus $t_{n}$ for each $k$, realize histograms of $\tau_{k}$ (if it does not depend on $t_{n}$ ) or obtain statistical characteristics of $\tau_{k}\left(t_{n}\right)$. As for $a_{k}\left(t_{n}\right)$, we can plot the magnitude and phase versus $t_{n}$ for each $k$, obtain the spectral characteristics (e.g. power spectrum) of $a_{k}\left(t_{n}\right), n=1,2, \ldots, N$ for each $k$, etc. We will see these possibilities in the next section.

These algorithms have been applied to a real ionospheric channel in the HF band (carrier frequency of $14.8 \mathrm{MHz}$ ), being the transmitter $1800 \mathrm{Km}$ apart from the receiver: the transmitter is in Madrid and the receiver is in the Canary Islands. The equivalent baseband channel has a bandwidth of $2700 \mathrm{~Hz}$ approximately, and the separation between two consecutive frequency samples is $37.5 \mathrm{~Hz}$. We point out that with a bandwidth of $2700 \mathrm{~Hz}$, it is only possible to get a delay resolution about 0.2 milliseconds in close rays of similar strength.

\section{Experimental results}

Consider a real ionospheric HF narrowband channel, and try to estimate the channel parameters. Suppose, we have a $M \times N$ matrix: $\hat{H}\left(\omega_{m} ; t_{n}\right), \quad m=1,2, \ldots, M ; n=1,2, \ldots, N$ of complex data (complex envelope), measured by an HF modem, and considering the multipath model, we can write

$$
\begin{gathered}
\hat{H}\left(\omega_{m} ; t_{n}\right) \approx \sum_{k=1}^{K} a_{k}\left(t_{n}\right) e^{-j \omega_{m} \cdot \tau_{k}\left(t_{n}\right)} \\
m=1,2, \ldots, M ; n=1,2, \ldots, N
\end{gathered}
$$

Our purpose is to estimate the number $K$ of ionospheric rays, the complex amplitudes $a_{k}(t)$ and the delays $\tau_{k}(t), k=1,2$, $\ldots, K$. from (13), considering the following parameter values: $M=73$ frequency samples, $N=5400$ time samples with a time rate of $33 \mathrm{~ms}$ (or an observation time of 3 minutes, approximately), and $\omega_{m}=2 \pi f_{m}$, with $f_{m}=f_{m-1}+37.5$, $m=1,2, \ldots, M$. A matrix of data $\hat{H}\left(\omega_{m} ; t_{n}\right), \quad m=1,2, \ldots, M$, $n=1,2, \ldots, N$, was measured repeatedly 12 times in one day (24 hours) and here we show the more interesting results. The ionospheric link was established between Madrid and the Canary Islands on 1st April 2008. Firstly, we have to say that at night (say, from local time 22:00 to local time 6:00, approximately) no link was possible (no path or ray was detected). In the day times, some paths or rays are possible: a strong ray and others slighter. In Figs. 1-4, we show two of these links: Figs. 1 and 2 correspond to a local time about 06:00, and Figs. 3 and 4 correspond to 12:00, approximately.

Fig. 1(a) shows delay realization sections of three possible paths, where estimation (median) delays are $\hat{\tau}_{1}=-0.02$, $\hat{\tau}_{2}=-0.33$ and $\hat{\tau}_{3}=0.52$ milliseconds (the strongest ray indicates the reference delay, i.e. $\hat{\tau}_{1} \approx 0$ ). Fig. $1(\mathrm{~b})$ shows delay histograms for all possible path estimations, and we can see up to five paths defined by the corresponding delays, although path numbers greater than three are not significant (according to the histogram, the number of detected cases is less than $10 \%$ for the fourth and the fifth paths). Fig. 2(a) shows coefficient-magnitude realizations of the three strongest paths (rays), where the strongest ray is about 10times the second strongest one and 20-times the third one. 

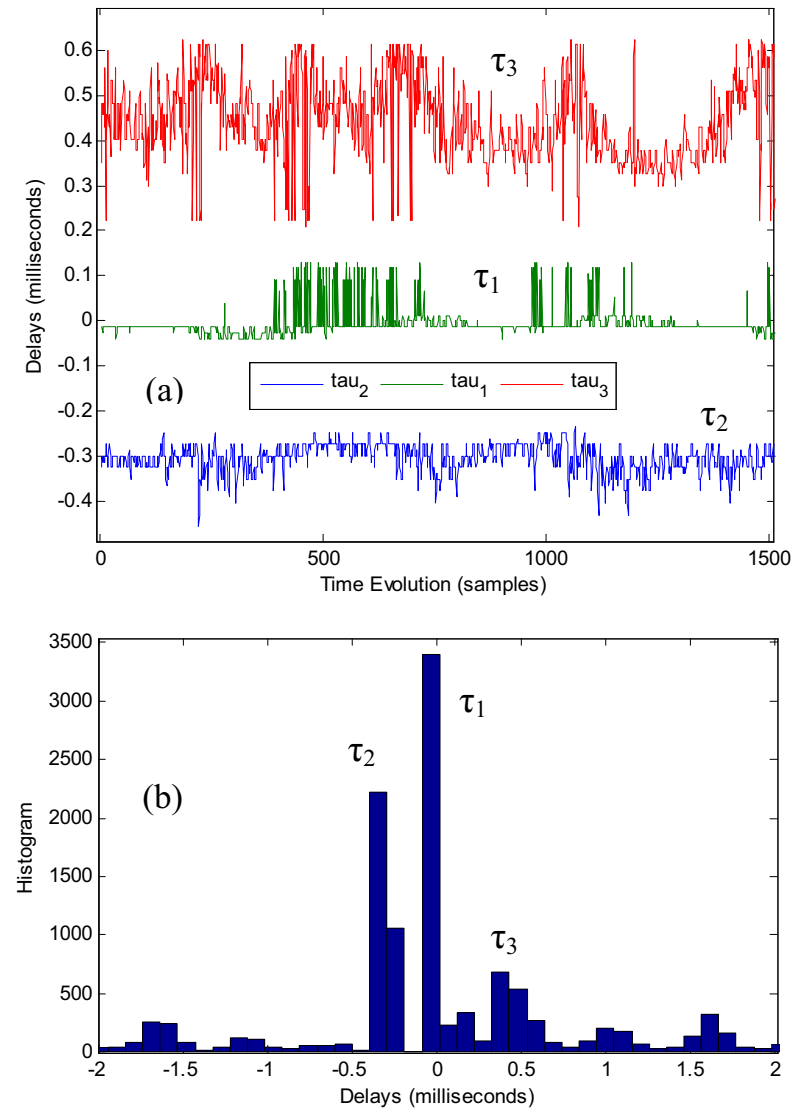

Fig. 1. Delay estimations for a real ionospheric channel. (a) Path-delay Estimations vs. Time Evolution (sample number) for the three strongest paths: $\tau_{k}(n), \mathrm{k}=1,2$ and 3 for $1<n<1500$. (b) Delay Histograms for 3400 samples of the delay sequences. The measurements were realized on 1st April at 6:00 a.m. for 3 minutes.

The estimated signal-to-noise ratio (SNR) for each coefficient is as follows: $\operatorname{SNR}\left(a_{1}\right)=17 \mathrm{~dB}, \operatorname{SNR}\left(a_{2}\right)=-3 \mathrm{~dB}$ and $\operatorname{SNR}\left(a_{2}\right)=$ $-8 \mathrm{~dB}$, approximately. In Fig. 2(b), we show estimations of the power spectral density for ray coefficients, where we have used a sequence of 3400 samples (4 sections of 850 samples each one). All curves are bell-shaped with approximately a mean Doppler frequency close to $-0.1 \mathrm{~Hz}$ (modem receiver tunes automatically the mean received frequency) and Doppler-frequency spread close to $0.1 \mathrm{~Hz}$. Finally, according to our measured results, the total power is about 12.1, and the powers of three rays are approximately: $P_{1}=11.7, P_{2}=0.1$ and $P_{3}=0.03$, if we remove all the ray signals from the received signal (13) it remains a flat power spectrum that corresponds to noise and interference with approximately a power of 0.2 units.

Fig. 3(a) shows delay realizations of four possible paths, where the median delays are $\hat{\tau}_{1}=-0.01, \quad \hat{\tau}_{2}=0.45$, $\hat{\tau}_{3}=-0.26$ and $\hat{\tau}_{4}=0.92$ milliseconds. Fig. 3(b) shows delay
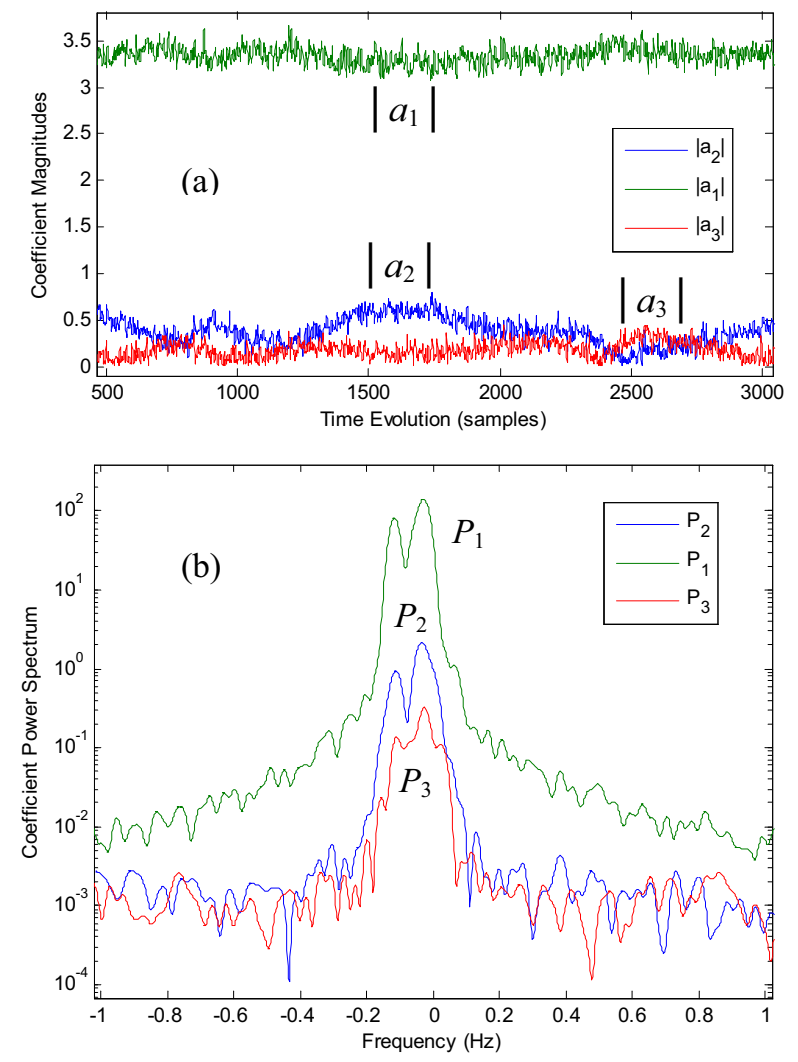

Fig. 2. Random coefficient realizations and power spectra for the real ionospheric channel of Fig 1. (a) Coefficient Magnitude Estimations vs. Time Evolution (sample number) for the three strongest paths: $\left|a_{k}(n)\right|, \mathrm{k}=1,2$ and 3 , for $500 \leq n \leq 3000$. (b) Power spectral density estimations $\left(P_{1}, P_{2}\right.$ and $\left.P_{3}\right)$ of the coefficients.

histograms for all possible path estimations, and we can see clearly the four significant paths defined by the corresponding delays. In Fig. 4(a) it is shown coefficient-magnitude realizations of the four strongest paths (rays), where the strongest ray is about 5-times the second strongest one, 10times the third strongest one and, also, 10-times the fourth one. The estimated signal-to-noise ratio (SNR) for each coefficient is approximately as follows: $\operatorname{SNR}\left(a_{1}\right)=15 \mathrm{~dB}$, $\operatorname{SNR}\left(a_{2}\right)=3 \mathrm{~dB}, \operatorname{SNR}\left(a_{3}\right)=-5 \mathrm{~dB}$ and $\operatorname{SNR}\left(a_{4}\right)=-4 \mathrm{~dB}$. In Fig. 4(b), we show estimations of the power spectral density for ray coefficients, where we have used a sequence of 3400 samples (4 sections of 850 samples each one). All curves are bell-shaped with approximately $-0.4 \mathrm{~Hz}$ mean Doppler frequency (negative value) and a Doppler-frequency spread close to $0.2 \mathrm{~Hz}$. The total power is about 12 , and the powers of four significant rays are approximately: $P_{1}=10.8$, $P_{2}=0.52, P_{3}=0.09$ and $P_{4}=0.1$, if we remove all the ray signals from the received signal (13) it remains a flat power spectrum that corresponds to noise and interference with approximately a power of 0.3 units. 

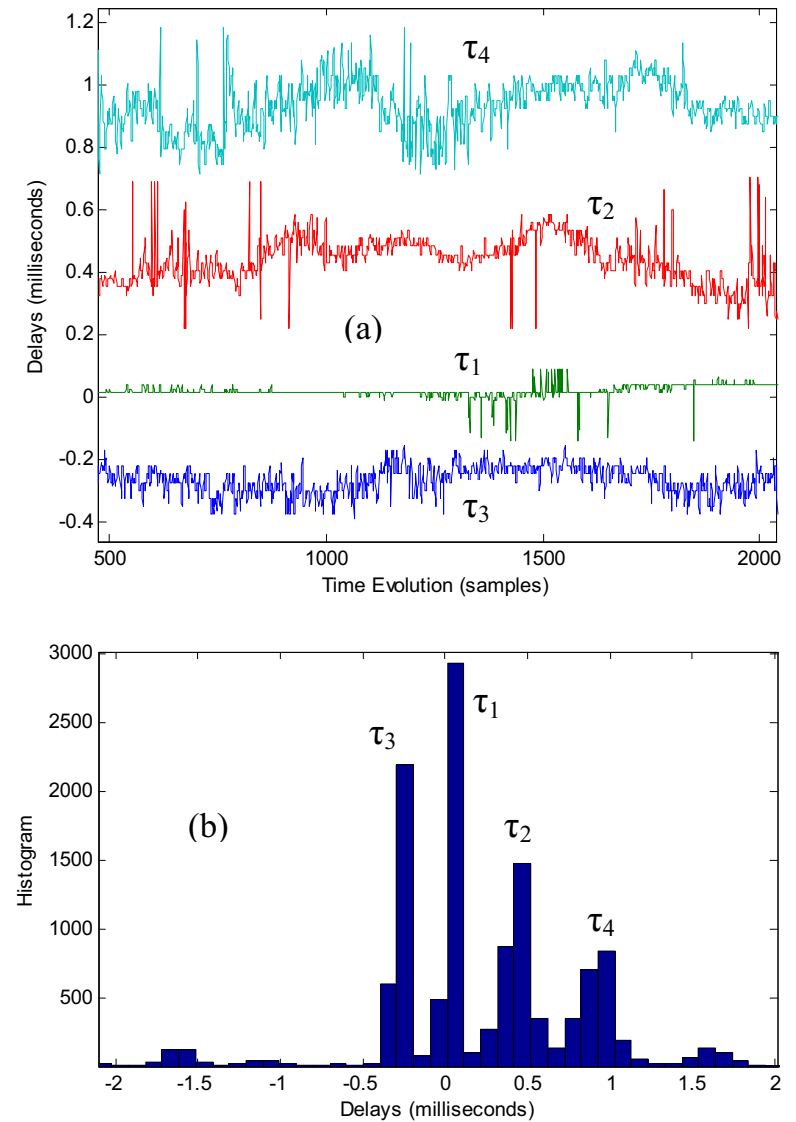

Fig. 3. Delay estimations for a real ionospheric channel. (a) Path-delay Estimations vs. Time Evolution (sample number) for the four strongest paths: $\tau_{k}(n), k=1,2,3$ and 4, for $500<n<2000$. (b) Delay Histograms for 3400 samples of the delay sequences. The measurements were realized on 1st April at 12:00 a.m. for 3 minutes.

\section{Conclusions}

We have described algorithms for estimating path delays and path coefficients. Our algorithms have been applied to parameter estimations of real ionospheric channels, showing high ability in ray detection, and in delay and coefficient estimations for each ray. This model cannot distinguish between ordinary and extraordinary propagation modes.

\section{Acknowledgements}

This work has been partially supported by the "Dirección General de Investigación" of the "Ministerio de Educación y Ciencia" under Grant TEC2007-67520-C02-01/TCM, and the "Universidad Politécnica de Madrid".

\section{References}

[1] C. C. Watterson, J. R. Juroshek, and W. D. Bensema. "Experimental Confirmation of an HF Channel Model", IEEE Trans. Communication,18 (6), pp.792-803, (1970).

[2] M. Leconte, and M. Testard. "A model of high frequencies (H.F) channel used to design a modem of
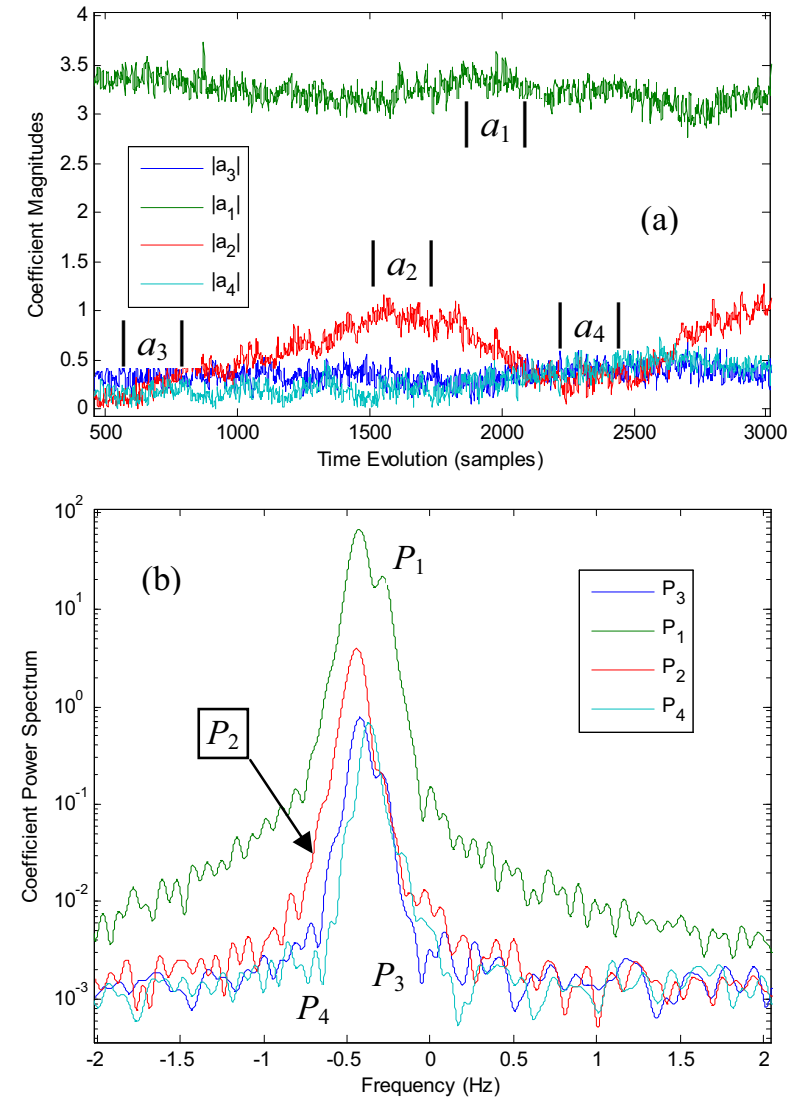

Fig. 4. Random coefficient realizations and power spectra for the real ionospheric channel of Fig 3. (a) Coefficient Magnitude Estimations vs. Time Evolution (sample number) for the four strongest paths: $\left|a_{k}(n)\right|, k=1,2,3$ and 4, for $500 \leq n \leq 3000$. (b) Power spectral density estimations $\left(P_{1}, P_{2}, P_{3}\right.$ and $\left.P_{4}\right)$ of the coefficients.

9600 bits/s rate in $3 \mathrm{kHz}$ of bandwidth". Proceedings IEEE Military Communications Conference (MILCOM) Vol. 1, pp. 351-355, (1997).

[3] J. F. Mastrangelo, J. J. Lemmon, L. E. Vogler, J. A. Hoffmeyer, L. E. Pratt, and C. J. Behm. "A New Wideband High Frequency Channel Simulation System". IEEE Transactions on Communications, 45 (1), pp. 26-34 (1997).

[4] V.E. Gherm, N.N. Zernov, and H.J. Strangeways. "Wideband HF simulator for multipath ionospherically reflected propagation channels". Proceedings of 12 th International Conference on Antennas and Propagation (ICAP 2003), Vol. 1, pp. 128-131 (2003).

[5] Mitra, S.K., and Kaiser, J.F. Handbook for Digital Signal Processing. New York: John Wiley \& Sons, (1993).

[6] Proakis, J.G. Digital Communications. New York: McGraw-Hill, Inc. (3rd Ed.), (1995).

[7] P. A. Bello. "Evaluation of Mobile Ultra Wideband Modems in Dense Multipath-Part 1: Channel Model" IEEE Transactions on Wireless Communications, 6 (11), pp. 4145-4153 (2007). 